\title{
Intestinal atresia following chemotherapy, presenting as superior mesenteric artery syndrome: A case report
}

\author{
XING WANG $^{1 *}$, JIAN-ZHONG LI ${ }^{1 *}$, YING-HUI YANG ${ }^{1}$, XIAO-LI HUANG ${ }^{1}$, YU WANG $^{2}$ and BIN WU ${ }^{1}$ \\ ${ }^{1}$ Department of Gastroenterology, The Third Affiliated Hospital of Sun Yat-sen University, Guangzhou, Guangdong 510630; \\ ${ }^{2}$ Department of Medical Oncology, Sun Yat-sen University Cancer Center, Guangzhou, Guangdong 510060, P.R. China
}

Received January 19, 2017; Accepted July 24, 2017

DOI: $10.3892 / \mathrm{mco} .2017 .1353$

\begin{abstract}
Multi-agent chemotherapy is recognized as the most common and effective treatment for Burkitt lymphoma, and intestinal mucosal injury is a common gastrointestinal complication following intensive chemotherapy. The aim of the present study was to describe a case of non-Hodgkin lymphoma with intestinal obstruction after chemotherapy in a young adult. The patient presented with aggravated vomiting during the second session of chemotherapy, which was initially attributed to superior mesenteric artery syndrome. However, following surgical intervention, the symptom was proven to be due to extreme intestinal stenosis in the ascending part of the duodenum. The patient underwent duodenojejunectomy and end-to-side anastomosis, and he recovered well from the operation. Although intestinal perforation and hemorrhage induced by chemotherapy have been previously reported sporadically, to the best of our knowledge, this is the first case report of distal duodenal obstruction due to intestinal atresia induced by polychemotherapy for lymphoma. We herein analyze the possible underlying reasons for the intestinal atresia and review the clinical and pathological characteristics of previously published relevant studies. The present findings may be helpful for increasing clinical awareness of this type of complication, as well as improving the management of patients treated with cytotoxic chemotherapeutic agents.
\end{abstract}

\section{Introduction}

Burkitt lymphoma is a highly aggressive type of B-cell non-Hodgkin lymphoma (NHL) and is characterized by

Correspondence to: Professor $\mathrm{Bin} \mathrm{Wu}$, Department of Gastroenterology, The Third Affiliated Hospital of Sun Yat-sen University, 600 Tian He Road, Guangzhou, Guangdong 510630, P.R. China

E-mail: wubin6@mail.sysu.edu.cn

${ }^{*}$ Contributed equally

Key words: intestinal obstruction, non-Hodgkin lymphoma, chemotherapy, atresia, superior mesenteric artery syndrome a translocation involving the c-MYC oncogene. Burkitt lymphoma is internationally recognized to comprise three clinical variants, namely endemic, sporadic and immunodeficiency-related. The endemic type is associated with Epstein-Barr virus infection and is most prevalent in Africa and the Middle East; the sporadic type occurs throughout the rest of the world and constitutes 1-2\% of adult and $30-40 \%$ of childhood NHL cases in Europe and North America; the immunodeficiency-related type is associated with infection by the human immunodeficiency virus $(1,2)$. Due to its chemosensitive nature, specific regimens of intensive chemotherapy have been developed for the treatment of Burkitt lymphoma, and a regimen of cyclophosphamide, vincristine, doxorubicin, high-dose methotrexate ifosfamide, etoposide and high-dose cytarabine (CODOX-M/IVAC) has been established as the standard therapy with a relatively high event-free survival (EFS) and overall survival (OS), particularly in patients aged $<65$ years (2). Moreover, several studies have reported that adding anti-CD20 monoclonal antibody (rituximab) to the therapy may achieve superior outcomes compared with controls. The CODOX-M/IVAC regimen has been reported to be associated with 2-year OS rates of $64-82 \%$ and an EFS rate of $92 \%(2,3)$.

Intestinal atresia affects neonates in the majority of the cases. Primary small intestinal atresia mostly affects the duodenum ( $>50 \%$ ), and is usually associated with chromosomal anomalies, such as trisomy 21 , as well as other factors, including race, maternal age and birthweight (4). Secondary atresia rarely occurs after necrotizing enteritis, intussusception, meconium peritonitis and abdominal surgeries (5). Although chemotherapy-induced intestinal mucositis has been broadly reported, and intestinal hemorrhage and spontaneous perforation have been considered as complications of NHL following intensive chemotherapy in children, intestinal atresia secondary to chemotherapy is uncommon (6-9). We herein describe a case of NHL in a young adult who developed extreme intestinal stenosis, likely triggered by chemotherapy, but presenting as superior mesenteric artery syndrome (SMAS).

\section{Case report}

A 22-year-old male patient presented to a community hospital with a 4-month history of fatigue, paleness and intermittent 
melena in July 2015. The patient reported suffering from anorexia for 1 month prior to the onset of the abovementioned symptoms. The patient had no history of hair coloring and no exposure to radioactive substances or oil-based paints. The white blood cell count was $17.5 \times 10^{9} / 1$ (normal range, $4.0-10.0 \times 10^{9} / 1$ ) and the hemoglobin level was $55 \mathrm{~g} / 1$ (normal range, $120-160 \mathrm{~g} / \mathrm{l})$. Baseline abdominal computed tomography (CT) detected multiple intestinal lesions in the epigastric and umbilical region, with extensive retroperitoneal lymphadenopathy. Biopsy of the descending part of the duodenum revealed the presence of diffuse large B-cell lymphoma. One month later, the patient presented with cramping pain and abdominal bloating, with edema of the bilateral lower limbs, and was transferred to a tertiary care hospital for further assessment. On physical examination, severe anemic appearance was noted. No superficial lymph nodes were palpated, and the findings of the cardiovascular and pulmonary examinations were unremarkable. There was a slight protuberance over the abdomen, but there was no tenderness or organomegaly.

The patient received one course of chemotherapy with rituximab, vincristine, epirubicin, etoposide, dexamethasone and cyclophosphamide (R-EPOCH regimen). The definitive diagnosis after the first course was Burkitt lymphoma and the patient received cyclophosphamide, doxorubicin, rituximab and vincristine, with intrathecal injection of cytarabine and methotrexate (modified CODOX-M regimen with rituximab). During the period of chemotherapy, the patient presented with repeated vomiting, which was relieved by administration of antiemetic drugs. On January 4, 2016, sudden onset of aggravated vomiting ensued, with palpitations and weakness of the limbs, which could not be relieved by antiemetic therapy. The patient reported no epigastric pain, abdominal distension or headache, but on physical examination, tenderness over the epigastric region and positive succussion splash were noted. The subsequent esophagogastroduodenoscopy (EGD) detected a large amount of fluid retention in the gastric cavity and the duodenum. There was no residual lymphoma or stenosis observed in the descending part of the duodenum detected on EGD (Fig. 1). Abdominal plain X-ray and alimentary tract radiography with barium revealed duodenal obstruction, raising the suspicion of superior mesenteric artery syndrome (Figs. 2 and 3). Ultrasound revealed a narrow angle of $10^{\circ}$ between the superior mesenteric artery and the abdominal aorta. The patient was primarily diagnosed with superior mesenteric artery syndrome and treated with proton pump inhibitors, somatostatin, nasogastric drainage and sufficient parenteral nutritional supply, but the symptoms continued. On February 21, 2016, the patient was transferred to the Department of Surgery for exploratory laparotomy. During surgery, a focal 'band-like' narrowing was observed at the junction between the ascending part of the duodenum and the proximal part of the jejunum, with a gross appearance of occluded intestinal lumen at the site of lesion. Of note, mild dilation was observed in the part of the duodenum posterior to the superior mesenteric artery (Fig. 4). Duodenojejunectomy and end-to-side anastomosis were performed during the surgery. The histopathology of the resected specimen revealed atrophy of the epithelium and severe structural disturbance of the submucosa and muscularis propria at the site of atresia (Fig. 5).

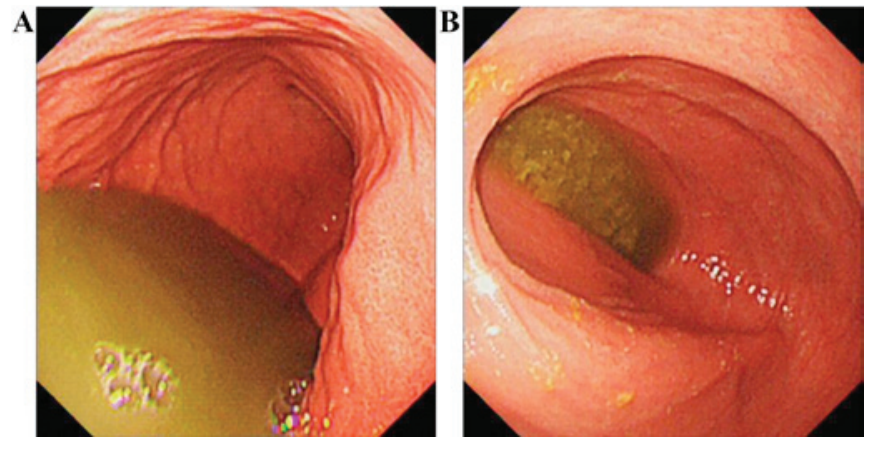

Figure 1. Findings on esophagogastroduodenoscopy: A large amount of fluid retention was observed in (A) the gastric body and (B) the descending part of the duodenum. No stenosis or neoplasms were identified.

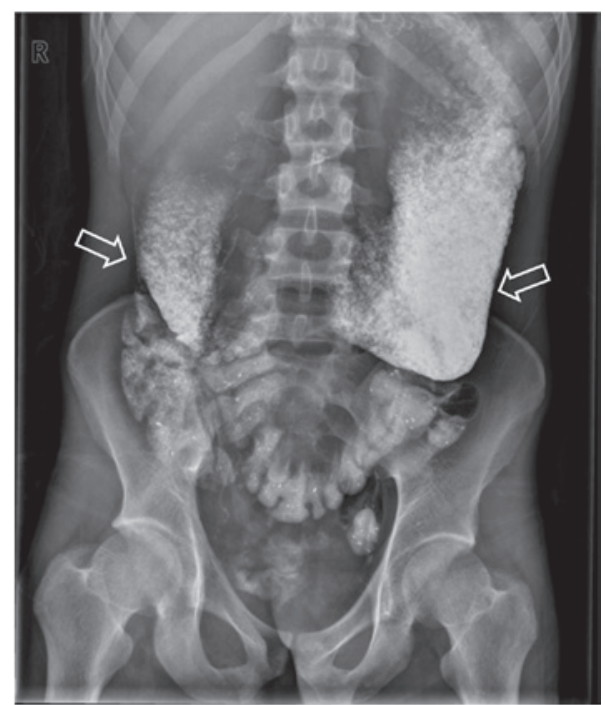

Figure 2. Plain abdominal X-ray. At 7 days after the previous barium radiography, residual barium remained mainly in the stomach and duodenal bulb (arrows).

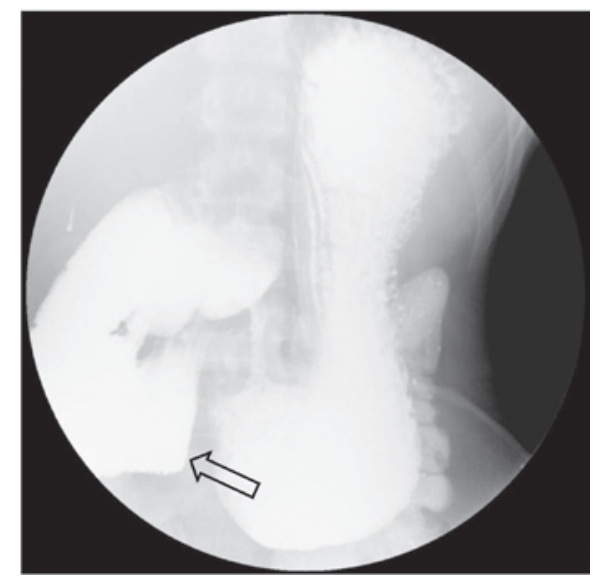

Figure 3. Barium radiography of the upper gastrointestinal tract: The barium filling was interrupted at the horizontal (3rd) part of the duodenum. A sharply vertical margin was noted as the compression of the superior mesenteric artery (arrow).

The patient recovered well from the procedure, with resolution of the symptoms, and further chemotherapies were well-tolerated. 

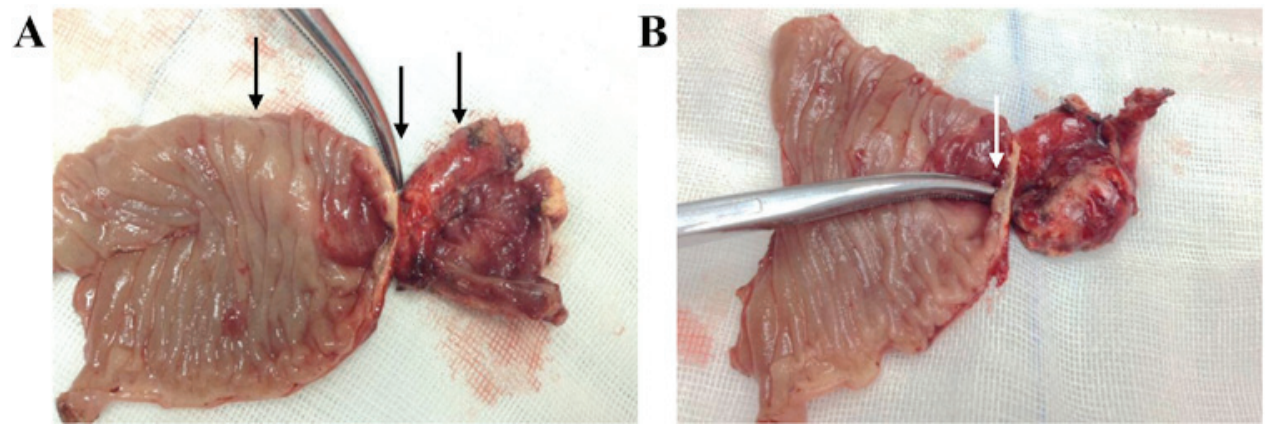

Figure 4. Macroscopic view of the resected intestine: The specimen exhibited a markedly thickened intestinal wall at the obstruction site in the junction of the duodenum and jejunum. (A) The left, middle and right arrows indicate the proximal dilated part, the site of the atresia and the distal part, respectively. (B) The apparent obstruction of the intestinal lumen was identified with a forceps exploration (arrow).
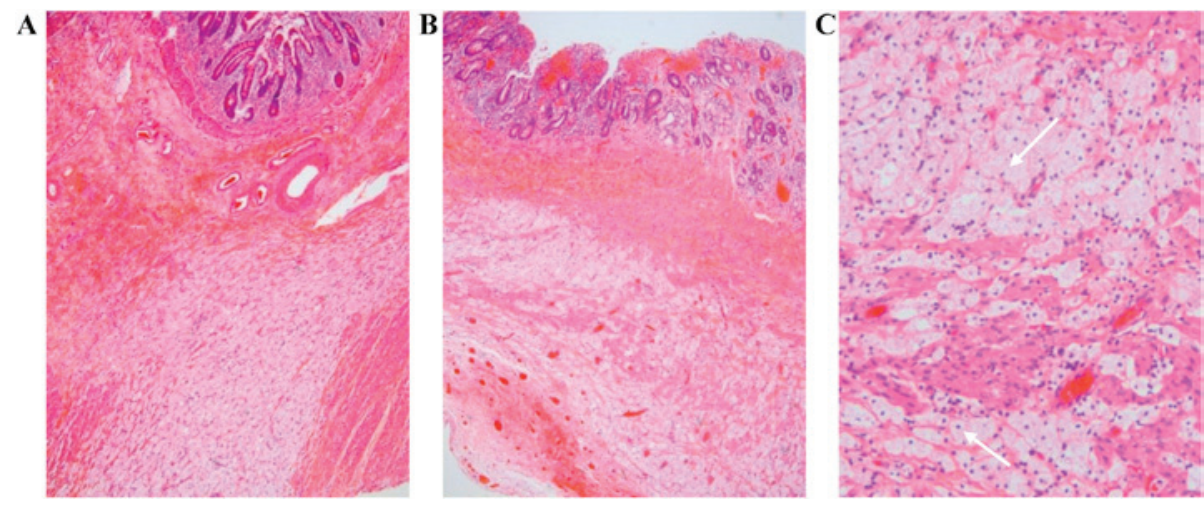

Figure 5. Histological examination of the site of the atresia: (A and B) Low-power magnification (x50) of the site of the atresia. Twisted villi and atrophy of the mucosal epithelium are observed. Low-level fibrosis was observed in the submucosa, with a large number of foam-like cells interrupting the muscularis propria. (C) High-power magnification (x200) of the submucosa. The muscularis propria of the site of the atresia is interrupted by foam-like cells (arrow). There is no evidence of neoplasia.

Written informed consent was obtained from the patient authorizing use and disclosure of his protected health information.

\section{Discussion}

We herein describe a case of secondary intestinal atresia, possibly induced by chemotherapy, in a young adult diagnosed with Burkitt lymphoma. The case is interesting, as the onset and progression of the symptoms were highly suggestive of SMAS. Although the present case included lymphomatous involvement of the duodenum prior to treatment, the microscopic examination of the surgical specimen detected no signs of neoplasia after chemotherapy.

Nausea, vomiting and diarrhea are most common gastrointestinal complications of cancer chemotherapy, and mucositis due to the damage of the proliferating cells at the base of the mucosal squamous epithelia or in the intestinal crypts usually involves the gastrointestinal tract (10). Hemorrhage and perforation of the small intestine have been reported in the previous literature as complications associated with chemotherapy for NHL; however, in the majority of the cases, evidence of the tumor is found on histological examination of the specimen. Coward et al reported a case of suspected chemotherapy-induced bowel obstruction in small-cell lung cancer, in which pathological examination of the excised bowel specimen revealed severe ulceration, transmural necrosis and extensive fibrosis (6). Kehoe et al reported that $>100$ patients developed intestinal obstruction following intraperitoneal chemotherapy and concluded that the majority of the obstructions were associated with the progression of the primary malignancy, whereas only $12 \%$ of the patients developed mechanical obstruction due to adhesions. The adhesions were described as 'coating or encasing the bowels' intraoperatively, but no apparent stenosis of the intestine was observed (7).

Several factors may contribute to the intestinal obstruction after chemotherapy. The administration of antiemetic drugs of 5-HT receptor antagonists may greatly decrease peristalsis and result in paralytic ileus; furthermore, constipation may occasionally develop secondary to prolonged bed rest. However, tumor cell necrosis under chemotherapy is hypothesized to be a prominent factor in the development of intestinal obstruction. Microtubular toxins, such as vincristine, are commonly associated with autonomic bowel dysfunction, and etoposide, as a podophyllotoxin derivative that interferes with microtubular binding, may cause paralytic ileus (6). Burkitt lymphoma as a highly proliferative hemotological malignancy usually involving several organs. The tumor cells infiltrating the wall of the small intestine are destroyed during chemotherapy, causing inflammation, necrosis, fibrosis of the adjacent tissue, and even perforation and hemorrhage $(9,11,12)$. In addition, it is widely known that chemotherapy may interrupt the DNA synthesis of intestinal mucosal cells and cause atrophy of epithelia (13). The transmural necrosis and fibrosis were not 
prominent on histological examination in the present case, but extensive tissue degeneration was observed in the submucosa and muscularis propria, with an extensive tissue cell response. The necrosis of lymphoma cells occurred in the submucosa and muscularis propria, and the space was replaced by large numbers of foam-like cells, which were likely derived from macrophages. Therefore, it is possible that the intestinal atresia observed in this case is attributable to the altered structure of the intestinal wall.

The diagnosis of SMAS is primarily based on clinical suspicion, with additional upper gastrointestinal barium examination, ultrosonography and/or CT angiography. The diagnosis is most probable when radiography detects a decreased aortomesenteric angle $\left(<25^{\circ}\right)$ and an aortomesenteric distance of $<10 \mathrm{~mm}(14,15)$. The case was misdiagnosed prior to surgery, since the clinical manifestations with acute weight loss, and the supportive findings on barium contrast examination, with an extremely narrow angle between the superior mesenteric artery and abdominal aorta, constituted strong evidence supporting the diagnosis of SMAS. Furthermore, the short distance between the predicted compression site of the superior mesenteric artery and the actual site of obstruction, also contributed to the misdiagnosis. Unfortunately, abdominal CT scan, which may have enabled identification of the exact obstruction site prior to surgery, was not performed in this case. However, regardless of intestinal necrosis, atresia or perforation, surgical intervention is considered to be a necessary intervention.

In summary, the present study describes a case of NHL, with post-chemotherapeutic intestinal atresia originally misdiagnosed as SMAS. This case indicates the importance of an abdominal CT scan in confirming the cause of the intestinal obstruction, and may also remind physicians to be aware of rare bowel complications associated with polychemotherapeutic regimens, and take measures for the protection of the intestinal mucosa during chemotherapy.

\section{References}

1. Molyneux EM, Rochford R, Griffin B, Newton R, Jackson G, Menon G, Harrison CJ, Israels T and Bailey S: Burkitt's lymphoma. Lancet 379: 1234-1244, 2012.
2. Casulo $\mathrm{C}$ and Friedberg J: Treating Burkitt lymphoma in adults. Curr Hematol Malig Rep 10: 266-271, 2015.

3. Wasterlid T, Brown PN, Hagberg O, Hagberg H, Pedersen LM, D'Amore $F$ and Jerkeman M: Impact of chemotherapy regimen and rituximab in adult Burkitt lymphoma: A retrospective population-based study from the Nordic Lymphoma Group. Ann Oncol 24: 1879-1886, 2013.

4. Takahashi D, Hiroma T, Takamizawa S and Nakamura T: Population-based study of esophageal and small intestinal atresia/stenosis. Pediatr Int 56: 838-844, 2014.

5. Yu KC, Wu XJ, Feng JX and Wei MF: Experienes of managing children with acquired intestinal stenosis and atresia. Chin J Pediatr Surg 36: 211-214, 2015 (In Chinese).

6. Coward JI, Ding NL, Feakins R, Kocher H, Popat S and Szlosarek PW: Chemotherapy-induced bowel obstruction in small cell lung cancer: A case report. Med Oncol 29: 2623-2625, 2012.

7. Kehoe SM, Williams NL, Yakubu R, Levine DA, Chi DS Sabbatini PJ, Aghajanian CA, Barakat RR and Abu-Rustum NR: Incidence of intestinal obstruction following intraperitoneal chemotherapy for ovarian tubal and peritoneal malignancies. Gynecol Oncol 113: 228-232, 2009.

8. Hata S, Pietsch J and Shankar S: Intestinal complications in children undergoing chemotherapy for mediastinal non-Hodgkin's lymphoma. Pediatr Hematol Oncol 21: 707-710, 2004.

9. Fallon SC, Redell MS, El-Bietar J, Lopez ME, Vasudevan SA and Brandt ML: Intestinal perforation after treatment of Burkitt's lymphoma: Case report and review of the literature. J Pediatr Surg 48: 436-440, 2013

10. Sausville EA and Longo DL: Principles of cancer treatment. In: Harrison's principles of internal medicine. Longo DL and Fauci AS (eds). McGraw-Hill Companies, New York, NY, pp708-709, 2011.

11. Howarth GS, Tooley KL, Davidson GP and Butler RN: A non-invasive method for detection of intestinal mucositis induced by different classes of chemotherapy drugs in the rat. Cancer Biol Ther 5: 1189-1195, 2006.

12. Ara C, Coban S, Kayaalp C, Yilmaz S and Kirimlioglu V: Spontaneous intestinal perforation due to non-Hodgkin's lymphoma: Evaluation of eight cases. Dig Dis Sci 52: 1752-1756, 2007.

13. Abel E, Ekman T, Warnhammar E, Hultborn R, Jennische E and Lange S: Early disturbance of microvascular function precedes chemotherapy-induced intestinal injury. Dig Dis Sci 50: 1729-1733, 2005.

14. Pottorf BJ, Husain FA, Hollis HJ and Lin E: Laparoscopic management of duodenal obstruction resulting from superior mesenteric artery syndrome. JAMA Surg 149: 1319-1322, 2014.

15. Merrett ND, Wilson RB, Cosman P and Biankin AV: Superior mesenteric artery syndrome: Diagnosis and treatment strategies. J Gastrointest Surg 13: 287-292, 2009. 